\title{
In situ observation of triple junction motion during recovery of heavily deformed aluminum
}

\author{
Yu, Tianbo; Hughes, Darcy A.; Hansen, Niels; Huang, Xiaoxu
}

Published in:

Acta Materialia

Link to article, DOI:

10.1016/j.actamat.2014.12.014

Publication date:

2015

Document Version

Peer reviewed version

Link back to DTU Orbit

Citation (APA):

Yu, T., Hughes, D. A., Hansen, N., \& Huang, X. (2015). In situ observation of triple junction motion during recovery of heavily deformed aluminum. Acta Materialia, 86, 269-278.

https://doi.org/10.1016/i.actamat.2014.12.014

\section{General rights}

Copyright and moral rights for the publications made accessible in the public portal are retained by the authors and/or other copyright owners and it is a condition of accessing publications that users recognise and abide by the legal requirements associated with these rights.

- Users may download and print one copy of any publication from the public portal for the purpose of private study or research.

- You may not further distribute the material or use it for any profit-making activity or commercial gain

- You may freely distribute the URL identifying the publication in the public portal

If you believe that this document breaches copyright please contact us providing details, and we will remove access to the work immediately and investigate your claim 
Structural pinning of triple junction motion during recovery of heavily deformed aluminum

Tianbo $\mathrm{Yu}^{\mathrm{a}, *}$, Darcy A. Hughes, Niels Hansen ${ }^{\mathrm{a}}$ and Xiaoxu Huang ${ }^{\mathrm{a}}$

${ }^{a}$ Danish-Chinese Center for Nanometals, Section for Materials Science and Advanced Characterization, Department of Wind Energy, Risø Campus, Technical University of Denmark, DK-4000, Roskilde, Denmark

Tianbo $\mathrm{Yu}^{*}$ :

Tel: (45) 46774706

Fax: (45) 46775758

Email: tiyu@dtu.dk

Darcy A. Hughes ～Email: darcyahughes@gmail.com

Niels Hansen: $\quad$ Email: niha@dtu.dk

Xiaoxu Huang: $\quad$ Email: xihu@dtu.dk

*Corresponding author. 


\begin{abstract}
:
Microstructural evolution during in situ annealing of heavily cold rolled aluminum has been studied by transmission electron microscopy (TEM) confirming that an important recovery mechanism is migration of triple junctions formed by three lamellar boundaries (Y-junctions). The migrating triple junctions are pinned by deformationinduced interconnecting and lamellar boundaries, which slow down the recovery process and lead to a stop-go migration pattern. This pinning mechanism stabilizes the deformation microstructure, i.e. the structure is stabilized by balancing driving and pinning forces controlling the rate of triple junction motion. Thereby recovery and the following recrystallization are strongly retarded. The underlying mechanisms are characterized and analyzed herein.
\end{abstract}

Keywords: Aluminum; Annealing; Deformation structure; Transmission electron microscopy (TEM); Triple junction; 


\section{Introduction}

Strengthening of metals by plastic deformation is of universal interest focusing today on deformation to high strains to produce materials with ultrafine structures and high strength. The strength has its origin in a high concentration of structural defects exemplified by narrowly spaced low and high angle deformation-induced boundaries $[1,2,3,4]$. These boundaries together with other defects raise the stored energy and the driving pressure for boundary migration, reducing the thermal stability of the strong materials $[5,6,7,8]$. For example, high purity $\mathrm{Al}(\geqslant 99.99 \%)$ deformed by torsion to various strains in liquid nitrogen was reported to recover and recrystallize below room temperature $[9,10]$, and high purity $\mathrm{Cu}(99.96 \%)$ deformed by equal channel angular extrusion (ECAE) to a high strain was reported to be partially recrystallized after long term storage at room temperature [11]. It has also been found, however, that the thermal stability can be relatively high with the addition of some impurities. For example, only a slight decrease in the hardness was observed when commercial purity $\mathrm{Al}(99.2 \%)$ was annealed at $100{ }^{\circ} \mathrm{C}$ after deformation by accumulative roll bonding (ARB) to a large strain [8], and heavily cold-rolled $\mathrm{Al}(99.5 \%)$ remained at the onset of recrystallization after annealing for 10 minutes at $300{ }^{\circ} \mathrm{C}$ (above 0.6 in terms of the homologous temperature) [12]. Such a stabilization may have its cause in the retardation of boundary migration by solute drag and particle pinning $[13,14,15,16]$. Other stabilizing methods may be found and utilized, in addition to stabilizing by solutes and particles, by examining the fundamental recovery and pinning mechanisms inherent in the structure of heavily deformed metals. Pinning interactions may depend on the properties of the deformation-induced boundaries, but it may also depend on their morphology. For example, it has been observed that migration of one type of triple junctions is an 
important recovery mechanism in heavily deformed aluminum $[17,18]$, leading to removal of boundaries and coarsening of a lamellar microstructure prior to recrystallization.

The structural morphology of highly strained metals is typically characterized by the presence of lamellar boundaries and interconnecting boundaries, which subdivide the structure. Based on their origins, these boundaries have been classified as geometrical necessary boundaries (GNBs) and incidental dislocation boundaries (IDBs), respectively $[1,19]$. The misorientation angle of GNBs increases more rapidly than that of IDBs during deformation, and after a high strain, the GNBs are typically of mediumto-high angle $\left(>5^{\circ}\right)$ and the IDBs are typically of low angle $\left(<5^{\circ}\right)[1,2]$. In this structure the triple junctions were classified into three categories based on their morphology: those joining three lamellar boundaries were classified as Y-junctions; the other two are $\mathrm{H}-$ and r-junctions which involve the interconnecting boundaries [17]. Among the three types, Y-junctions moved during recovery annealing. Their motion usually involved one to three high angle boundaries $\left(>15^{\circ}\right)$, and the misorientation angle of the extending boundary was not necessarily larger than those of the receding boundaries (Yu et al. 2011). Thus the situation is different from early considerations of triple junction motion as a recovery mechanism, where two low angle dislocation boundaries zip up (Li 1960; Li 1966; Clauer et al. 1970). In heavily deformed samples (Yu et al. 2011), it was also observed that migration $\mathrm{Y}$-junctions interacted strongly with the surrounding deformation structure and that these Y-junctions appeared to be pinned when meeting interconnecting and lamellar boundaries. Such a pinning mechanism is important not only fundamentally but also technologically, as it may retard or inhibit Y-junction motion and thereby increase the stability of strongly deformed metals and alloys. In the 
present study we have chosen to use in situ annealing of samples in a transmission electron microscope in order to study the mechanisms underlying migration and pinning of Y-junctions in heavily deformed aluminum.

\section{Experimental details}

Commercial purity aluminum AA1050 (99.5\% purity with an initial grain size of $\sim 100$ $\mu \mathrm{m})$ was cold rolled to true strains of 2, 4 and 5.5 at room temperature, where the smallest strain $\varepsilon=2$ is near the lower bound for a completely lamellar deformation structure. Since the lamellar boundaries are parallel to the rolling plane $[1,2]$, we choose the longitudinal section, containing the rolling direction (RD) and the normal direction (ND), for all transmission electron microscopy (TEM) observations. As a result, the quasi two-dimensional deformation structure is well represented in TEM thin foils, making it possible to observe characteristic structural changes taking place during annealing. The TEM foils were prepared using a modified window technique [20], and the thickness of thin areas for observation was about $0.2-0.3 \mu \mathrm{m}$. The in situ experiments were carried out in a JEM 2100 transmission electron microscope, which was operated at a relatively low accelerating voltage of $120 \mathrm{kV}$ in order to reduce radiation damage. TEM foils of deformed states were mounted to a double-tilt heating holder. After the deformation structure was characterized, the foil was heated to $100{ }^{\circ} \mathrm{C}$ followed by a holding period, and then the temperature was further increased in steps of $20{ }^{\circ} \mathrm{C}$ at a heating rate of $0.2-0.4{ }^{\circ} \mathrm{C} / \mathrm{s}$ with a holding period of $3-5$ minutes between two temperature increases. The maximum temperature reached was typically $300{ }^{\circ} \mathrm{C}$. During both heating and holding periods, the microstructural changes were recorded by a TVIPS FastScan camera with a maximum frame rate of 12 frames/s at full resolution. 
For some samples, the heating program was interrupted by cooling the sample to room temperature in order to better characterize the microstructure of intermediate states. A Kikuchi diffraction method [21] was used to determine the misorientation angles for boundaries of interest.

The quasi two-dimensional boundary structure can be well characterized in our TEM foils and the in-plane migration of $\mathrm{Y}$-junctions is driven by in-plane driving forces, so the effect of free surfaces can be considered to be small for a qualitative study of this motion. However, minor processes occurred due in these foils due to the free surfaces (Yu et al. 2012), and the kinetics of structural evolution is different from that in bulk interior [17]. In order to study the partially recovered structure in the bulk interior, $\mathrm{Al}$ following a strain of 5.5 was annealed at $180{ }^{\circ} \mathrm{C}$ for $1 \mathrm{~h}$, and the microstructure after annealing was examined in a JEM 2000FX transmission electron microscope, which was also operate at $120 \mathrm{kV}$.

\section{Results}

\subsection{In situ observations}

When annealed below $160{ }^{\circ} \mathrm{C}$, the deformation structure in the TEM foil was stable and no structural changes were directly noticed during in situ observation. After the annealing temperature was further increased, both dislocation activity and Y-junction motion coupled to local boundary migration became active. Most of the microstructural changes took place during short temperature increases, whereas only limited changes were observed during long temperature holding periods, in an agreement with the quasilogarithmic time dependence of recovery $[22,23]$. Both dislocation activity and Yjunction motion led to a decrease in the density of loose dislocations. Only Y-junction 
motion, however, changed the structural morphology. An overview of the structural change is shown in Fig. 1. The deformed lamellar structure (Fig. 1a) coarsened uniformly and transformed gradually into a more equiaxed structure (Fig. 1c) by migration of many $\mathrm{Y}$-junctions along the RD.
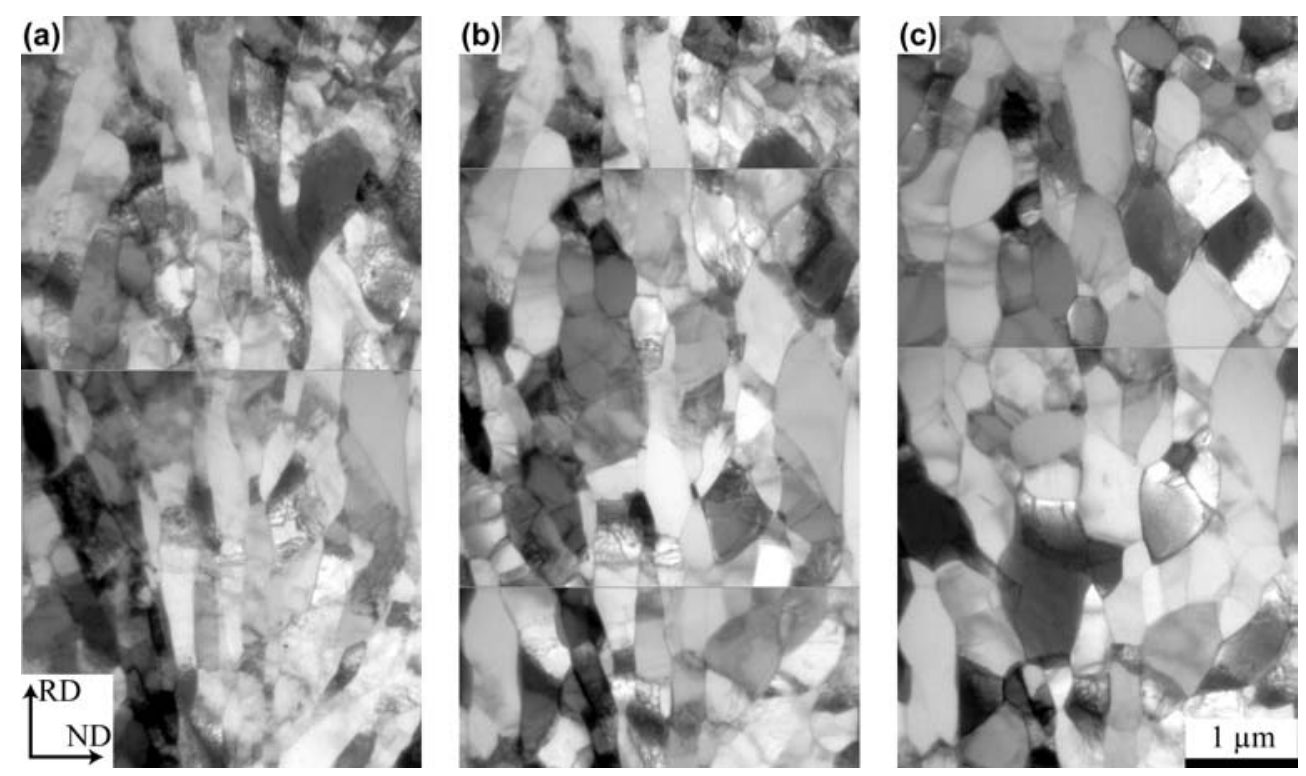

Figure 1. Gradual transformation of the lamellar deformation microstructure into a more equiaxed structure by $\mathrm{Y}$-junction motion during annealing. Microstructure in the longitudinal section of $\mathrm{Al}$ (a) cold rolled to a true strain of 2, (b) at the same region after in situ annealing in the microscope at highest $200^{\circ} \mathrm{C}$ for about 5 minutes and (c) after further annealing at highest $280{ }^{\circ} \mathrm{C}$ for about 5 minutes.

Moving Y-junctions strongly interacted with boundaries and dislocation structures encountered in their nearby vicinity. Fig. 2 shows a typical example in a lamellar structure (video in supplementary data). The Y-junction in the center of the micrograph (arrowed in Fig. 2a) was initially pinned by a neighboring interconnecting boundary, which was attached to one of the receding lamellar boundaries on the left side of the Y-junction. As the Y-junction migrated downwards (Fig. 2b), this interconnecting 
boundary was forced to extend and bow, exerting a large dragging force on the attached lamellar boundary. Four dislocations are visible in this interconnecting boundary, which is of tilt character and is sketched in Fig. $2 \mathrm{j}$. With further annealing, these four dislocations bowed successively and then were unpinned from the moving Y-junction one by one, i.e., as the bowing was released in each dislocation, that force unpinned the dislocation from the receding lamellar boundary, caused it to glide across the Y-junction and then come to rest on the extending lamellar boundary (Fig. 2b-i). For depinning of each dislocation, the incubation time was more than $10 \mathrm{~s}$ (e.g. $30.3 \mathrm{~s}$ from Fig. $2 \mathrm{~b}$ to Fig. 2c), but the glide of each dislocation only took less than $1 \mathrm{~s}($ e.g. $0.5 \mathrm{~s}$ from Fig. 2c to Fig. 2d). After all of the four dislocations in the interconnecting boundary were unpinned, the $\mathrm{Y}$-junction migrated further and then stopped near the next set of interconnecting boundaries. Note, the misorientation angle, $0.5^{\circ}$, of the interconnecting boundary remained the same during this process.

(a)

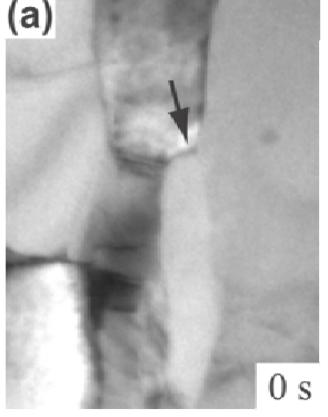

(e)

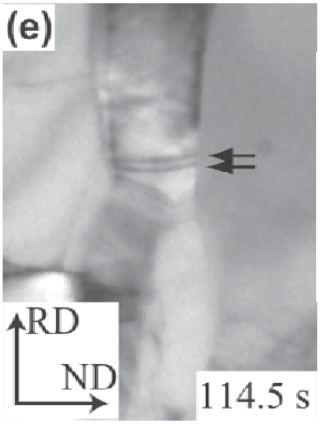

(b)

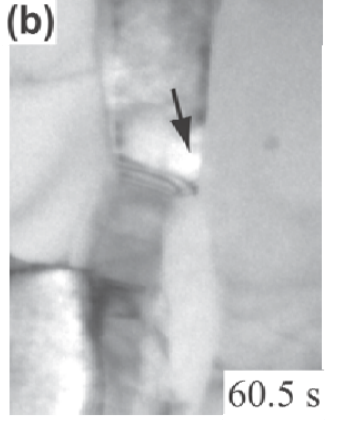

(f)

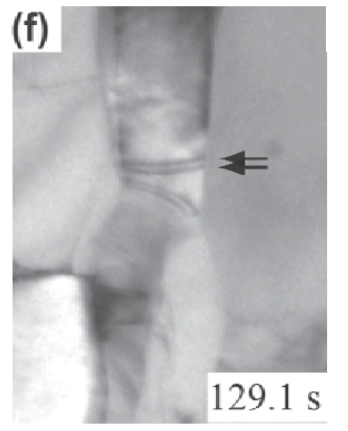

(c)
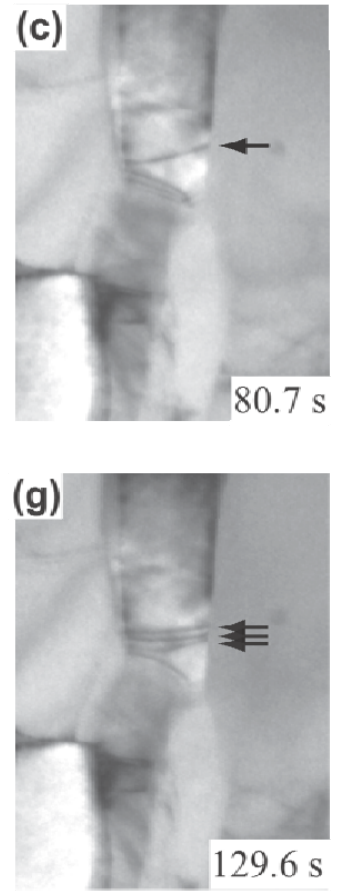

(d)

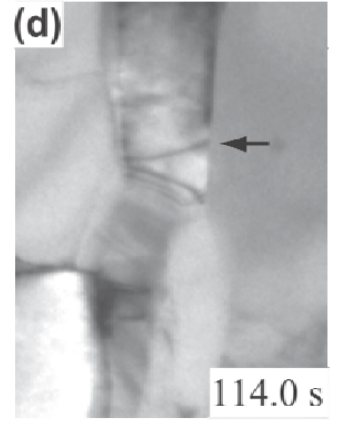

(h)

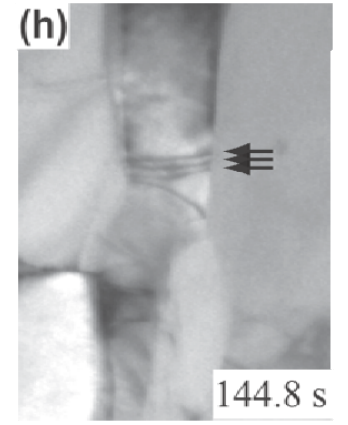

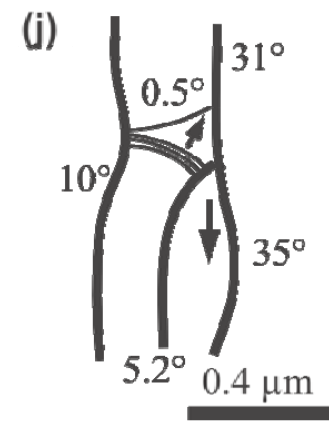

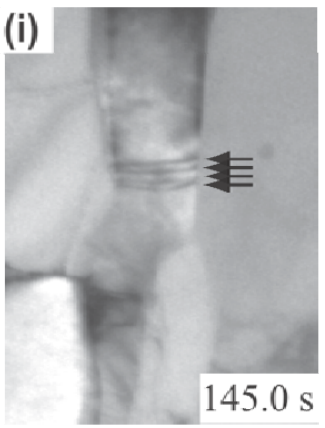


Figure 2. Migration of a Y-junction and its lengthy interaction with an attached interconnecting boundary in $\mathrm{Al}$ cold rolled to a true strain of 4 during annealing at 180 ${ }^{\circ} \mathrm{C}$. The time sequence from 0 to $145.0 \mathrm{~s}$ is shown in each micrograph. The arrows in (a) and (b) point to the Y-junction, whose motion was retarded by the attached interconnecting boundary; each small arrow in (c) to (i) points to an interconnecting boundary dislocation which was unpinned after bowing. In the corresponding sketch in (j), lamellar boundaries are shown in bold lines, and the interconnecting boundary (of tilt character with a misorientation angle $0.5^{\circ}$ ) is composed of four dislocations, which are shown in thin lines. In the sketch, the big arrow indicates the direction of Y-junction motion, whereas the small arrow indicates the direction of dislocation glide during depinning (video in supplementary data).

The interaction between the moving Y-junction and the dislocation structure shown in Fig. 2 occurred in a well-developed lamellar structure at a strain of 4 . At the lower strain of $\varepsilon=2$, the lamellar morphology was less well-developed in some regions, and additional interactions between a migrating $\mathrm{Y}$-junction and its surrounding microstructure were observed as shown in Fig. 3 (video in supplementary data). The Yjunction marked by an arrow in Fig. 3a was initially pinned by a neighboring interconnecting boundary on its left side (labeled as A in the sketch Fig. 3g). When the annealing temperature was increased, the $\mathrm{Y}$-junction moved slightly causing boundary A to bow and then unpin. This Y-junction escaped and moved up, leading to a local increase in the boundary spacing of two neighboring lamellae. Meanwhile, three dislocations (B) from interconnecting boundary A were still connected to the receding lamellar boundary (D), and therefore were forced to glide ahead of the migrating Y- 
junction as well as along boundary A (Fig. 3h) before meeting another lamellar boundary (C) on its left side. When meeting lamellar boundary $\mathbf{C}$, the Y-junction was temporarily pinned by this boundary on its left and an interconnecting boundary $(\mathbf{F})$ on its right (Fig. 3e); the three dislocations glided away from boundary $\mathbf{D}$ and onto this newly encountered lamellar boundary $\mathbf{C}$. Further annealing resulted in depinning and further migration of the Y-junction until it was pinned by another interconnecting boundary (E) as shown in Fig. 3f and sketched in Fig. 3i. When the Y-junction stopped, it had an increased dihedral angle and its lamella at $\mathbf{D}$ was wider (Fig. $3 \mathrm{f}$ and 3i). Misorientation measurements after cooling the sample showed that both lamellar and interconnecting boundaries involved have low misorientation angles (Fig. 3f), which are typical for many regions at this relatively low strain.
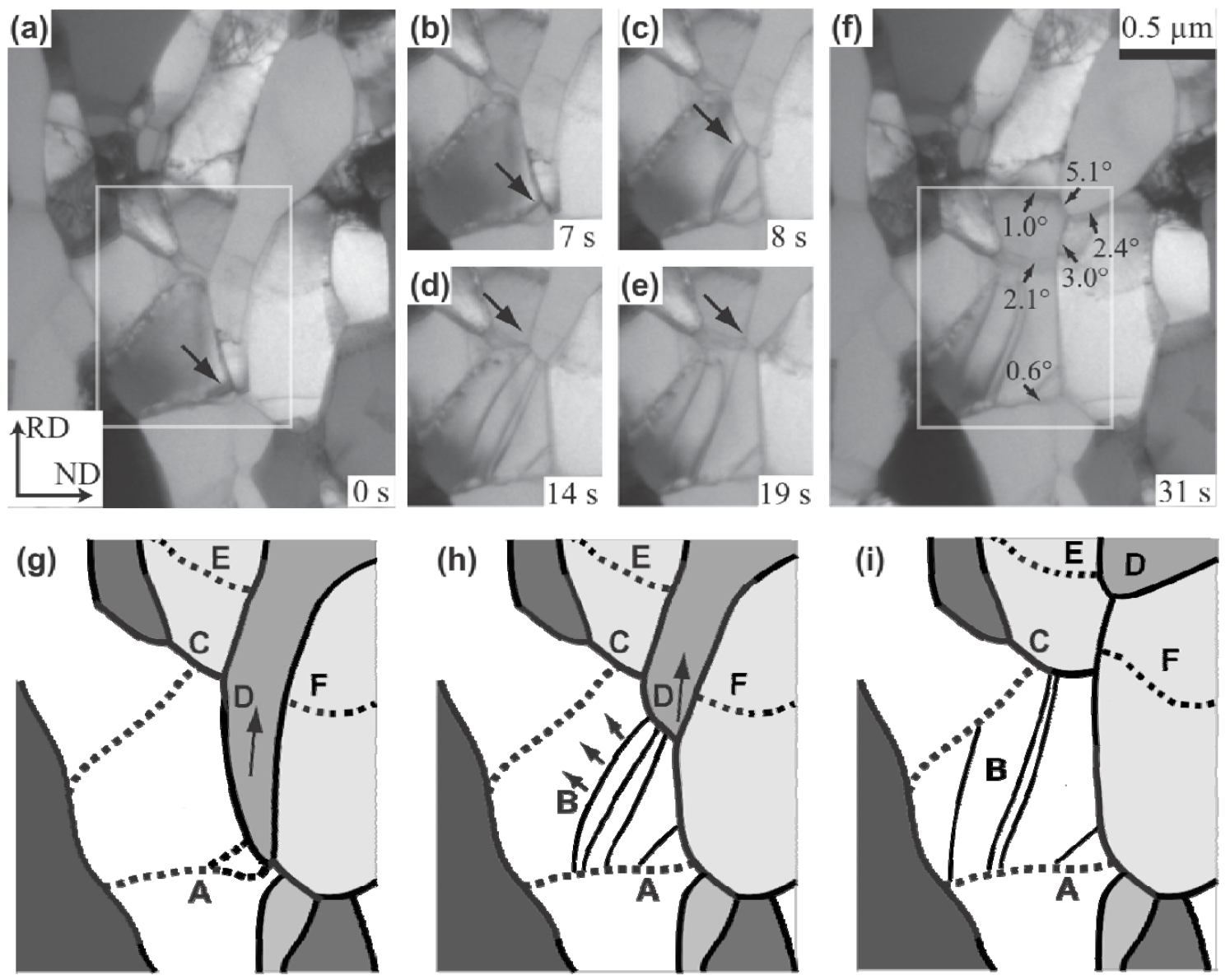
Figure 3. Migration of a $\mathrm{Y}$-junction and its interaction with surrounding microstructure in $\mathrm{Al}$ cold rolled to a true strain of 2 when the annealing temperature was increased from 260 to $280{ }^{\circ} \mathrm{C}$. The time sequence from 0 to $31 \mathrm{~s}$ is shown in each micrograph. The marked areas in (a) and (f) are the same as the areas shown in (b)-(e). The arrows in (a)-(e) point to the migrating $\mathrm{Y}$-junction with interconnecting boundary dislocations attached on its left side. The misorientation angles of associated boundaries are shown in (f). In the corresponding sketches before (g), during (h) and after (i) Y-junction motion, lamellar boundaries are shown in bold lines (e.g. C and D), interconnecting boundaries in broken lines (e.g. A and $\mathbf{E}$ ), and dislocations in thin lines (B) (video in supplementary data).

When viewed on the longitudinal section (RD-ND plane), the deformed lamellar structure is quasi two-dimensional since most of lamellar boundaries are close to an edge on position. As a consequence, $\mathrm{Y}$-junctions are typically parallel to the foil normal (the transverse direction, TD) and migrate roughly along the RD. However, occasionally structural changes along the TD were observed in thin TEM foils. Fig. 4 (video in supplementary data) shows an example, where the middle part of the dark lamella in Fig. 4a had an edge inside the foil, i.e. a Y-junction line parallel to the RD (see the inset in Fig. 4h). With increasing annealing temperature, this Y-junction bowed along the TD, cutting the dark lamella into two parts and creating two Y-junctions parallel to the foil normal (Fig. 4b and c). Upon further annealing, the newly created pair of Y-junctions (labeled as $\mathbf{B}$ and $\mathbf{C}$ in the sketch Fig. 4h) migrated away from each other along the RD, removing the original dark lamella while largely keeping the lamellar morphology. During this process, the migration of $\mathrm{Y}$-junctions $\mathbf{B}$ and $\mathbf{C}$ was retarded by attached 
lamellar and interconnecting boundaries, so the migration rate changed significantly as shown by the migration curves (distance versus time) in Fig. 5; whereas Y-junctions A and $\mathbf{D}$ were completely pinned by neighboring boundaries on both sides.
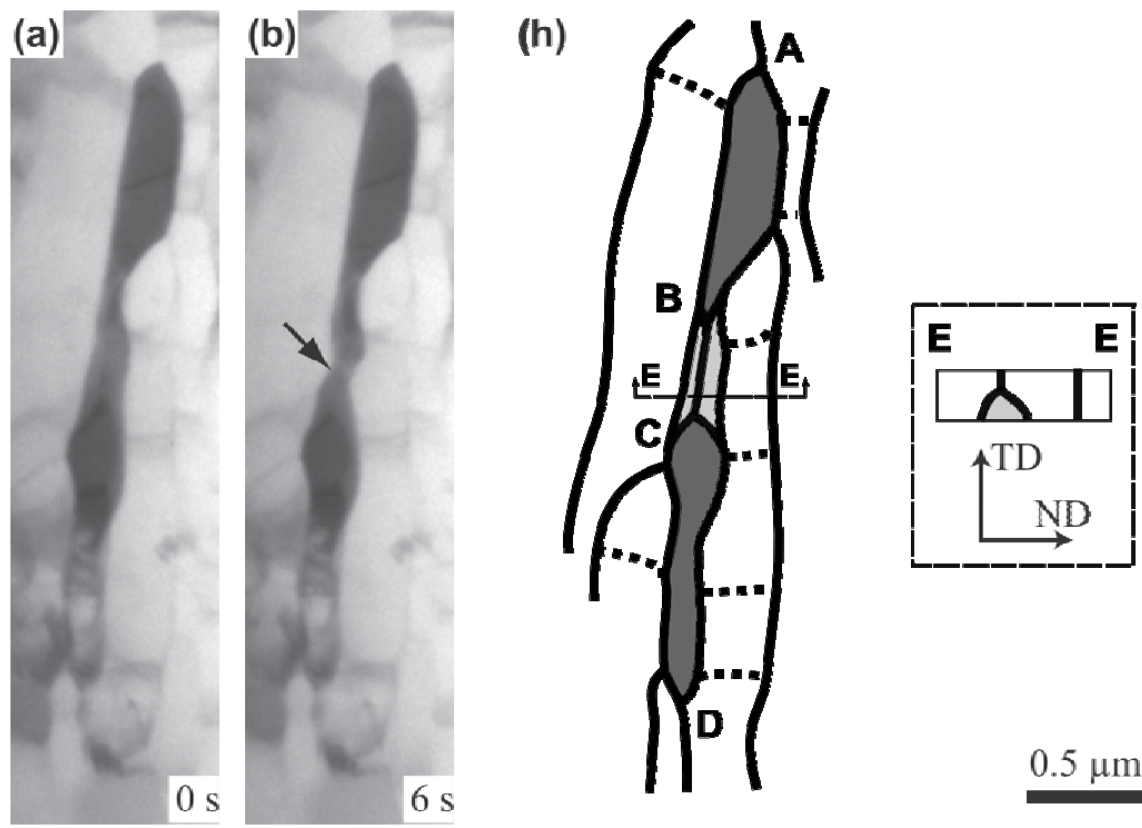

(c)

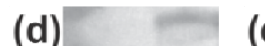

(e)
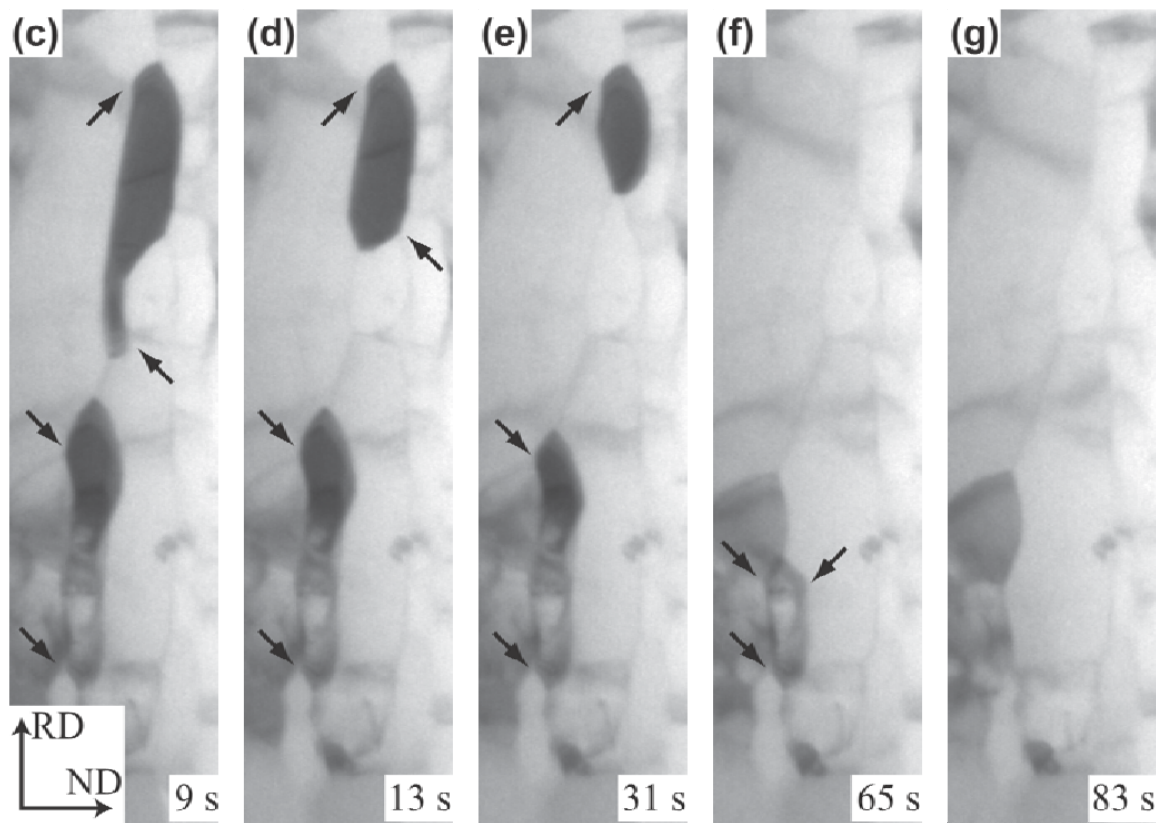

Figure 4. Break up and removal of a lamella by $\mathrm{Y}$-junction motion in Al cold rolled to a true strain of 4 when the annealing temperature was increased from 180 to $200^{\circ} \mathrm{C}$. The time sequence from 0 to $83 \mathrm{~s}$ is shown in each micrograph. The arrow in (b) indicates 
the location of break up due to Y-junction motion in the TD, and the arrows in (c) to (f) indicate pinning of Y-junction motion by attached boundaries. In the corresponding sketch in (h), lamellar boundaries are shown in solid lines, and interconnecting boundaries in broken lines. Four Y-junctions are labeled. The boundary structure in the transverse section E-E is sketched in the inset (video in supplementary data).

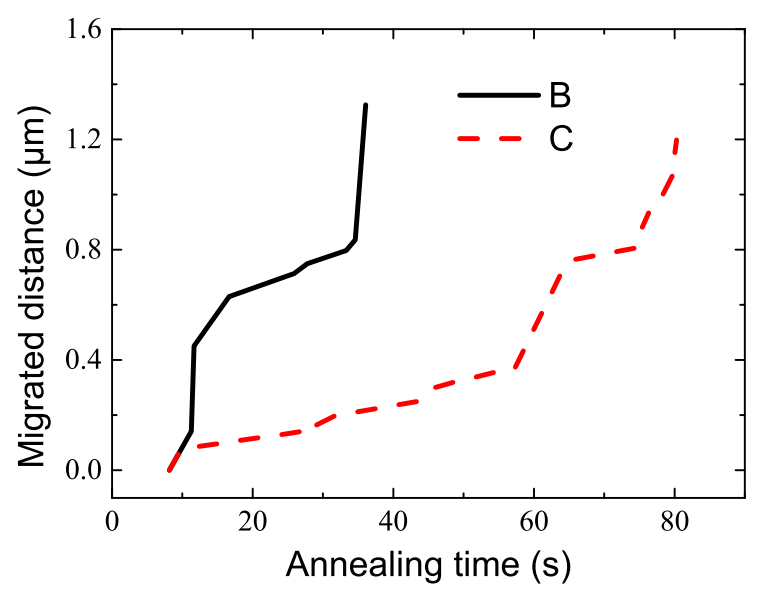

Figure 5. The distance migrated versus time of two $\mathrm{Y}$-junctions $\mathbf{B}$ and $\mathbf{C}$ shown in Fig. 4 during in situ annealing showing the change in slope as different boundaries are encountered and the junctions are pinned.

At higher annealing temperatures, Y-junctions migrated rapidly, but attached lamellar and interconnecting boundaries still had a strong pinning effect. Fig. 6 shows an example (video in supplementary data). In the first $4 \mathrm{~s}$, the arrowed Y-junction was pinned by a neighboring interconnecting boundary and the microstructure was unchanged (Figs. 6a and b). In the next $0.2 \mathrm{~s}$, the $\mathrm{Y}$-junction migrated downwards rapidly before stopped by two neighboring interconnecting boundaries (Fig. 6c). Afterwards, the Y-junction adjusted its position slightly, but still pinned by those two interconnecting boundaries on its both sides (Fig. 6d). 

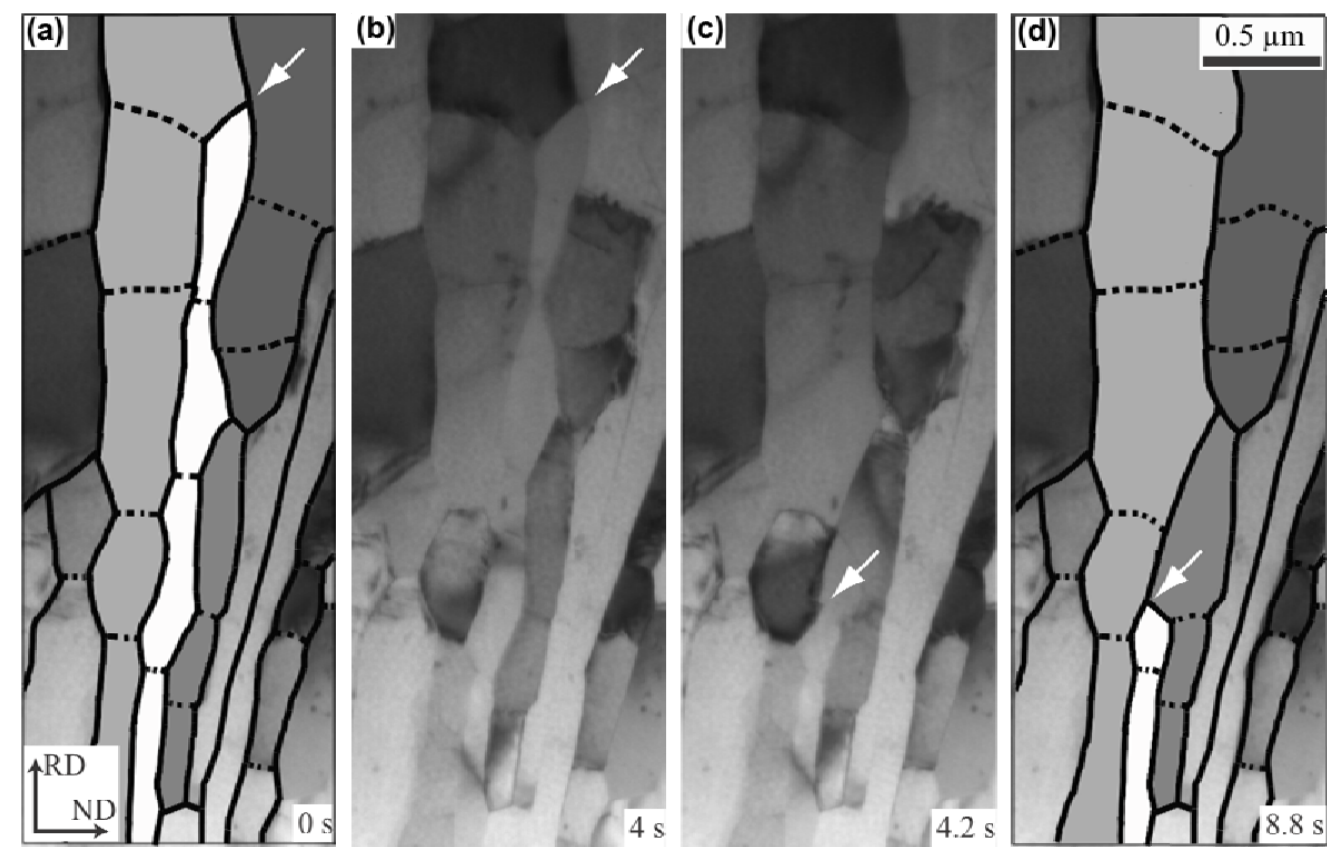

Figure 6. Rapid motion of a Y-junction (white arrows) at a higher annealing temperature of $\sim 320{ }^{\circ} \mathrm{C}$ and the subsequent arrest of that $\mathrm{Y}$-junction near two interconnecting boundaries (broken lines) in $\mathrm{Al}$ cold rolled to a true strain of 5.5. The time sequence from 0 to $8.8 \mathrm{~s}$ is shown in images (a) to (d). Images (a) and (d) are composite overlays of the boundary tracings and micrographs (video in supplementary data).

Globally, the lamellar structure coarsened uniformly during annealing; whereas locally Y-junction motion shortened and/or removed an individual lamella causing the adjacent lamellae to coarsen. The shortened/removed lamellae were typically thinner than the average lamellar boundary spacing, but occasionally thick lamellae were also observed to be shortened or even removed by Y-junction motion. The migrating Yjunctions interacted with the deformed microstructure in the neighborhood, and were frequently retarded or pinned by neighboring interconnecting and lamellar boundaries. 


\subsection{The partially recovered structure}

The partially recovered structure cut from the bulk interior is shown in Fig. 7a. It is clear that the lamellar morphology was retained although the lamellar boundary spacing had increased, in an agreement with the in situ observations. Compared to the deformed state, annealing at $180{ }^{\circ} \mathrm{C}$ for $1 \mathrm{~h}$ increased the average lamellar boundary spacing by almost $50 \%$ but caused little change in the average interconnecting boundary spacing (cell length, including all cells within each lamella), which increased from $0.78 \mu \mathrm{m}$ to $0.88 \mu \mathrm{m}$. Such a coarsening pattern supports that the dominant recovery process is $\mathrm{Y}$ junction motion, which removes lamellar boundaries but keeps interconnecting boundaries in retained lamellae.
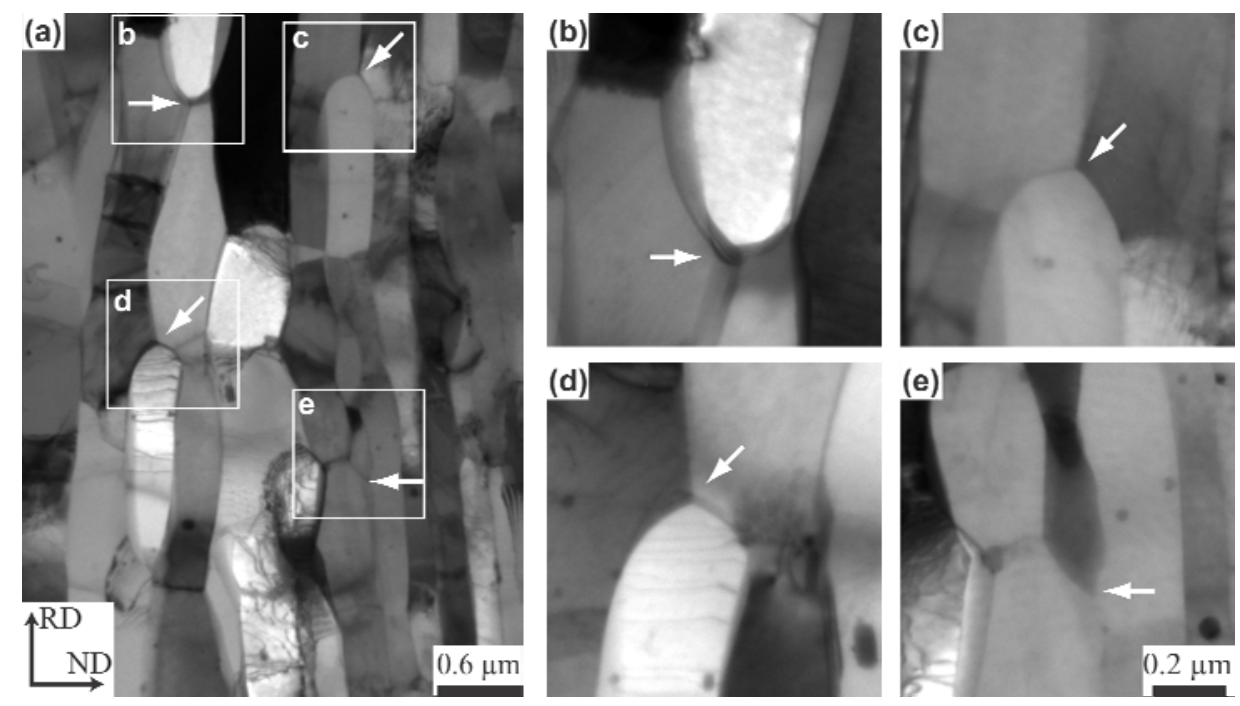

Figure 7. (a) Microstructure coarsened by bulk annealing at $180{ }^{\circ} \mathrm{C}$ for $1 \mathrm{~h}$ shown in the longitudinal section at a strain of 5.5 in Al. Stabilization of Y-junctions by neighboring boundaries is exemplified by the four marked regions that are magnified in (b)-(e), where the positions of Y-junctions are indicated by arrows. 
The partially recovered structure contains many $\mathrm{Y}$-junctions, and $4 \mathrm{Y}$-junctions within marked regions in Fig. 7a are magnified in Fig. 7b-e. A closer investigation of these Y-junctions revealed that they were typically in the vicinity of attached interconnecting or lamellar boundaries. These attached boundaries increased the dihedral angles at the $\mathrm{Y}$-junctions, thereby reducing the driving force for $\mathrm{Y}$-junction motion and stabilizing the partially recovered structure. The distribution of the projected distance from a $\mathrm{Y}$-junction to the nearest attached position, considering both sides and both interconnecting and lamellar boundaries, is shown in Fig. 8. The distribution gives an average distance of $94 \mathrm{~nm}$, whereas for a random distribution of Y-junctions, the average distance would be $1 / 3$ of the average cell length, i.e. $1 / 3 \times 880 \approx 293 \mathrm{~nm}$. Such a big difference indicates that $\mathrm{Y}$-junctions are not randomly distributed in the partially recovered structure. Instead, they form relatively stable configurations.

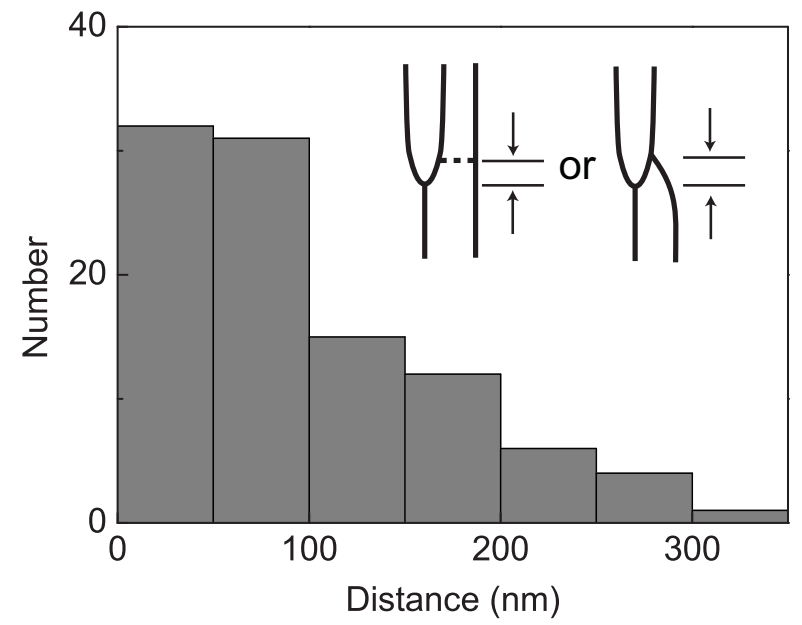

Figure 8. Distribution showing the very close association, $94 \mathrm{~nm}$ average, of the projected distance from a Y-junction to the nearest attached position of a stabilizing boundary at a strain of 5.5 in $\mathrm{Al}$ annealed at $180{ }^{\circ} \mathrm{C}$ for $1 \mathrm{~h}$. The distance was measured along the lamella as illustrated in the inset, where lamellar boundaries are shown in solid lines and an interconnecting boundary is shown in a broken line. 


\section{Discussion}

\subsection{Recovery in heavily deformed Al}

Recovery plays an important role in Al [23], consuming a large fraction of stored energy in samples deformed to large strains [8] prior to nucleation of recrystallization $[12,18]$. The present study is specifically directed towards recovery of samples deformed by cold rolling, introducing a fine scale lamellar microstructure. The microstructure is subdivided by extended lamellar boundaries [2], which typically are flat and stable. However in places where these lamellar boundaries meet, i.e. the Yjunctions, there is a concentration of high stored energy supplying the driving force for the migration of these junctions during recovery [17]. In the deformed structure, each lamella is divided by short interconnecting low angle boundaries (see Introduction). The current in situ study shows that the interconnecting boundaries are fairly stable and do not migrate during recovery annealing, although they interact with gliding dislocations and migrating Y-junctions and also adjust themselves, leading to annihilation of redundant dislocations and sharpening of the boundaries. Besides lamellar and interconnecting boundaries, loose dislocations are also present in the deformed structure, and their rearrangement and annihilation were shown to be important recovery mechanisms in the initial stage of recovery $[24,25]$. At later stages of recovery before recrystallization, $\mathrm{Y}$-junction motion overshadows other processes.

\subsection{Recovery by Y-junction motion}

Y-junction motion has been confirmed to be an important recovery mechanism in the current in situ study of heavily deformed Al. Y-junction motion replaces two lamellar 
boundaries by one and removes both dislocations and interconnecting boundaries within the removed lamella volume, while maintaining a lamellar structural morphology. Such a mechanism increases the average lamellar boundary spacing, and at the same time approximately maintains the average interconnecting boundary spacing in retained lamellae during early coarsening. After migration of a Y-junction, the thickness of a retained lamella (the width of lamella shown in TEM images taken in the longitudinal section) may be unchanged or increased due to the removal of its neighboring lamellae; the length of a retained lamella may be unchanged or decreased due to Y-junction motion (although the average length of retained lamellae may increase due to preferential removal of thin lamellae, which are also short in average). As a result, the aspect ratio of the structure decreases gradually, leading to a gradual transition from a lamellar to a more equiaxed morphology [18].

The driving force for $\mathrm{Y}$-junction motion comes from many sources, e.g. Yjunction line energy and strain energy from dislocations, but the principal source is boundary energy [17]. For a given Y-junction and its three lamellar boundaries, the migration depends on the dihedral angle and local curvature of two receding boundaries. These two parameters are often altered by attached lamellar and interconnecting boundaries, and the driving force can thus be changed during migration by these boundaries. Since the motion of a Y-junction always involves the local migration of boundaries, the boundary mobility is another important parameter. Y-junction motion may also have a misorientation dependence since high angle boundaries are supposed to have higher mobility than low angle boundaries [26]. However, experimental data (e.g. Fig. 3) clearly show that even Y-junctions formed by three low angle lamellar 
boundaries can also migrate, indicating other factors may selectively enhance Yjunction motion and the associated boundary mobility. For example, interconnecting boundaries within a lamella that are being removed by $\mathrm{Y}$-junction motion may increase the driving force for $\mathrm{Y}$-junction motion and accelerate the process.

Y-junction motion in deformed lamellar structures differs in many ways from normal grain growth after recrystallization in coarse-grained samples. The deformed structure contains a high stored energy, for example about $2 \mathrm{MJ} / \mathrm{m}^{3}$ in $\mathrm{Al}$ [8], and typically the driving force for Y-junction motion is one or two orders of magnitude larger than that for grain growth. During Y-junction motion, both the mobility of Yjunctions and the mobility of boundaries may control the overall kinetics, whereas during grain growth the kinetics may be controlled solely by the boundary mobility. Moreover, the apparent activation energy increases significantly from $110 \mathrm{~kJ} / \mathrm{mol}$ to 240 $\mathrm{kJ} / \mathrm{mol}$ during Y-junction motion in $\mathrm{Al}$ [18] in line with Kuhlmann-Wilsdorf's suggestion (Kuhlmann 1948), whereas during grain growth it is commonly found to be constant (e.g. [27]). On the other hand, Y-junction motion may be similar to the coarsening of nanocrystalline materials, where there is also a large driving force and where the kinetics is also affected by triple junction mobility (Gottstein and Shvindlerman 2006). However, the structural morphology is significantly different between two cases.

\subsection{Structural pinning of $\mathbf{Y}$-junction motion}

Interconnecting boundaries attached to a shortening lamella from the outside, i.e. interconnecting boundaries in neighboring lamellae (the first case shown in Fig. 8 inset), may exert pinning forces on Y-junction motion. The frequency of this close interaction 
and stationary pinning in the partially recovered structure was demonstrated in Figs. 7 and 8. The dynamic pinning of an interconnecting boundary during $\mathrm{Y}$-junction motion is illustrated in Fig. 9. The interconnecting boundary is pinned to the receding lamellar boundary, and bows following the migrating Y-junction. Since the interconnecting boundary resists both extending and depinning, extra energy is required to unpin the Yjunction from the interconnecting boundary. This energy may depend on many parameters.

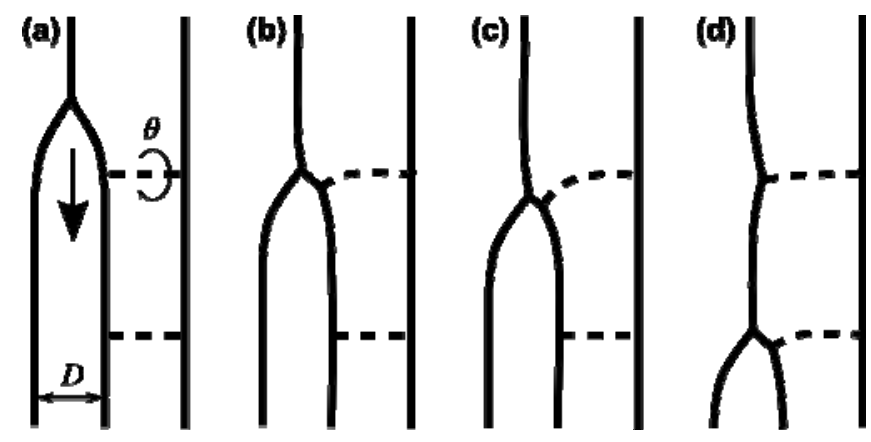

Figure 9. Schematic drawing that shows the pinning and depinning interactions between a moving Y-junction and interconnecting boundaries (dashed lines), including the bowing and slight extension of the interconnecting boundary during annealing. The arrow indicates the direction of $\mathrm{Y}$-junction motion, $D$ is the thickness of the shortening lamella and $\theta$ is the misorientation angle of the interconnecting boundary.

If only the initial and the final states are considered and the detailed interaction is ignored, then two important parameters are the energy per unit area of the interconnecting boundary and the thickness of the shortening lamella, which affects the extent to which the interconnecting boundary has to be extended. Consequently, the pinning effect from low angle interconnecting boundaries may increases with its misorientation angle since the boundary energy typically increases with increasing 
misorientation angle [28]. Moreover, the pinning effect from interconnecting boundaries may be larger in thick shortening lamellae than that in thin ones.

If the detailed interaction during depinning is considered, then other parameters may also affect the pinning force. For example, the character of the interconnecting boundary may play a role since tilt boundaries are easier to move than twist boundaries [29]. In the deformed lamellar structure, edge dislocations within interconnecting boundaries of tilt character are oriented to glide under the stress exerted by the motion of Y-junctions (see Fig. 10). So these dislocations may glide and escape during the depinning process (e.g. Fig. 3). Their depinning stress, consequently, may be lower than that of twist/mixed interconnecting boundaries. Moreover, the length of interconnecting boundaries may also play a role since long dislocations are easier to move than short ones (see Figs. 2 and 3). Interconnecting boundaries are typically short in high strain samples due to the small spacing of lamellar boundaries, so significant glide of dislocations from interconnecting boundaries following Y-junction motion may only occur after substantial coarsening of the microstructure (e.g. Fig. 3).

During the depinning process, interconnecting boundaries may develop a high curvature, and they may also have higher energies than those calculated by the ReadShockley equation [28] due to the deviation from a low energy dislocation structure. During depinning, the interconnecting boundary has to move across the Y-junction (Fig. 9), thereby temporarily forming a quadruple line. However, the interconnecting boundary may be not exact parallel to the Y-junction, so dislocations in the interconnecting boundary may bow and unpin sequentially instead of simultaneously, as observed in Fig. 2 and sketched in Fig. 10. 
In the structure after depinning, the interconnecting boundary is extended compared to the initial state, but the amount of extension may be smaller than that for regions without interconnecting boundaries, i.e. a cusp may be developed on the newly formed (extending) lamellar boundary under an energy balance (Fig. 9). Therefore during recovery annealing the interconnecting boundaries have a strong influence on the newly formed lamellar boundaries, leading to an undulating boundary (see Figs. 1c and 9d). In contrast this influence is slight when the interconnecting boundaries are newly formed by statistical trapping during deformation.
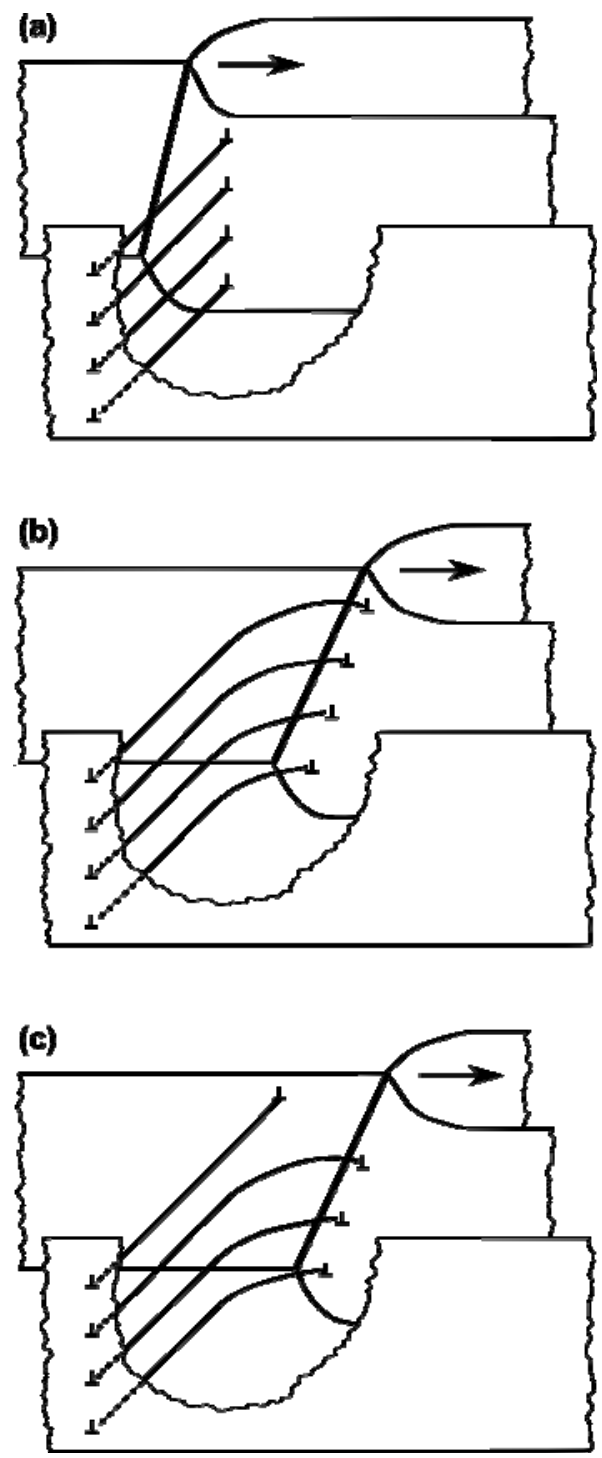
Figure 10. A 3D sketch showing pinning and depinning interactions between a moving Y-junction and individual boundary dislocations in an attached interconnecting boundary. The arrow indicates the direction of Y-junction motion. In (a) the interconnecting boundary exerts a drag force on boundary migration at the Y-junction. With increased $\mathrm{Y}$-junction motion in (b), interconnecting boundary dislocations glide, extend and bow sharply while attempting to maintain their boundary configuration. Bowing of the top and nearest dislocation reaches a critical point, releases and that dislocation is pushed across the Y-junction in (c).

Similarly, an attached lamellar boundary (the second case shown in Fig. 8 inset) may also exert a pinning force on Y-junction motion as illustrated in Fig. 11, although it may occur less frequently than pinning by interconnecting boundaries due to the length of a lamella. In this case, the thickness of the two lamellae may be the most important parameter determining the pinning effect; whereas the boundary energy varies marginally since lamellar boundaries are typically medium-to-high angle boundaries. When both of the two lamellae are thin, their contact area is small at the pinning position and depinning may be easier than that for thick lamellae.
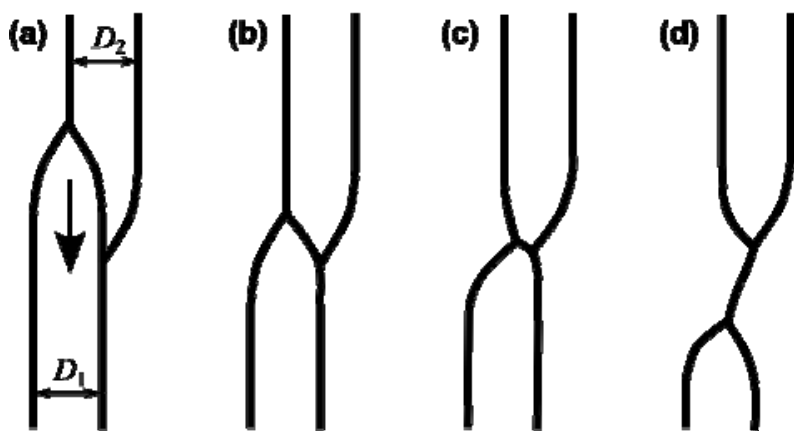
Figure 11. A sketch showing pinning and depinning interactions between a moving $\mathrm{Y}$ junction and an attached lamellar boundary. The arrow indicates the direction of Yjunction motion, and $D_{1}$ and $D_{2}$ are the thickness of lamellae.

The dependence on lamella thickness due to the pinning process is in good agreement with experimental results showing that thin lamellae are more likely to be removed [17] and that the activation energy for $\mathrm{Y}$-junction motion increases with increasing lamella spacing during recovery [18]. This dependence is also consistent with the high recovery rate observed in samples deformed to very high strains where the lamellar boundary spacing is small [8].

\subsection{Structural self-stabilization}

High strain deformation of Al introduces finely spaced interconnecting and lamellar boundaries. In the subsequent recovery annealing, the deformed microstructure coarsens via Y-junction motion, which is strongly retarded by the attached interconnecting and lamellar boundaries. Therefore, the effect of these deformation induced boundaries is paradoxical: on one hand they store deformation energy and provide the driving force for recovery and recrystallization, but on the other hand they stabilize the microstructure by retarding or pinning $\mathrm{Y}$-junction motion and boundary migration. Note that these stabilizing forces are maintained within the structure across the different strain levels examined. With increasing strain, the increase in stored energy and the refinement of lamellar boundaries are counterbalanced by the increase in pinning force due to the decreased spacing and increased misorientation angle of the interconnecting boundaries. The deformation structure is therefore self-stabilized against coarsening during recovery 
and nucleation of recrystallization. Self-stabilization from structural pinning is a new concept which parallels previously known stabilizing factors like solute drag and particle pinning $[13,14,15]$.

The current study has focused on Al, which has a high stacking fault energy (SFE). In metals with lower SFEs, e.g. $\mathrm{Ni}$ and $\mathrm{Cu}$, the deformation microstructure at large strain is also subdivided by interconnecting and lamellar boundaries, but the boundary spacings are significantly smaller than that in $\mathrm{Al}[1,30]$. In that case, the finer spacing of interconnecting boundaries may in turn provide a stronger structural pinning effect, balancing the effect of a larger driving force. In those metals, the density of loose dislocations between lamellar boundaries is also higher than that in $\mathrm{Al}$. Those loose dislocations may also be preferentially attached close to Y-junctions, stabilizing the microstructure as well as providing driving forces for restoration.

\section{Conclusion}

The microstructural evolution during annealing of heavily cold-rolled commercial purity aluminum (AA1050) has been studied by in situ TEM, and the structural pinning from lamellar and interconnecting boundaries on Y-junction motion has been analyzed. The following conclusions can be made.

1. Y-junction motion is an important recovery mechanism during annealing of heavily deformed commercial purity $\mathrm{Al}$, which has a finely spaced lamellar structure. The migration of Y-junctions causes shortening and removal of lamellae and local coarsening of neighboring lamellae. Thin lamellae are generally under higher driving force to be removed by Y-junction motion than thick lamellae. 
2. The interaction between moving Y-junctions and the lamellar and interconnecting boundaries they encounter, significantly alters the rate of Y-junction motion. The motion is either retarded through pinning and depinning processes due to local boundary structures, or completely stopped under a force balance.

3. During recovery annealing, the driving force is larger and the pinning force is smaller for thin lamellae than for thick ones. The tendency for thin lamellae to be removed by $\mathrm{Y}$-junction motion is therefore higher than that for thick ones, and the recovery rate is high in samples deformed to large strains having small lamellar boundary spacings and larger stored energies.

4. The deformation microstructure resulting from deformation to very large strains is subdivided by low and high angle boundaries, which can resist structural coarsening during annealing as well as providing driving forces for recovery and recrystallization. A balance of these forces enhances the structural stability of the deformed structure.

\section{Acknowledgement}

The authors gratefully acknowledge the support from the Danish National Research Foundation (Grant No. DNRF86-5) and the National Natural Science Foundation of China (Grant No. 51261130091) to the Danish-Chinese Center for Nanometals, within which this work has been performed.

\section{References}




\section{References}

[1] Hughes DA, Hansen N. Acta Mater. 2000;48:2985.

[2] Liu Q, Huang X, Lloyd DJ, Hansen N. Acta Mater. 2002;50:3789.

[3] Huang XX, Hansen N, Tsuji N. Science 2006;312:249.

[4] Zhang HW, Huang X, Hansen N. Acta Mater. 2008;56:5451.

[5] Doherty RD, Hughes DA, Humphreys FJ, Jonas JJ, Jensen DJ, Kassner ME et al. Mater.Sci.Eng.A-Struct.Mater.Prop.Microstruct.Process. 1997;238:219.

[6] Driver JH. Scr.Mater. 2004;51:819.

[7] Humphreys FJ, \& Hatherly M. Recrystallization and related annealing phenomena. Pergamon Pr, 2004.

[8] Cao WQ, Godfrey A, Hansen N, Liu Q. Metall.Mater.Trans.APhys.Metall.Mater.Sci. 2009;40A:204.

[9] Schmidt J, Haessner F. Z.Phys.B-Condens.Mat. 1990;81:215.

[10] Haessner F, Schmidt J. Acta Metall.Mater. 1993;41:1739.

[11] Mishin OV, Godfrey A. Metall.Mater.Trans.A-Phys.Metall.Mater.Sci. 2008;39A:2923.

[12] Mishin OV, Godfrey A, Jensen DJ, Hansen N. Acta Mater. 2013;61:5354.

[13] Smith CS. Trans. AIME 1948;175:15.

[14] LUCKE K, DETERT K. Acta Metallurgica 1957;5:628.

[15] CAHN J. Acta Metallurgica 1962;10:789.

[16] Ferry M, Hamilton NE, Humphreys FJ. Acta Mater. 2005;53:1097.

[17] Yu T, Hansen N, Huang X. Proc.R.Soc.A-Math.Phys.Eng.Sci. 2011;467:3039.

[18] Yu T, Hansen N, Huang X. Acta Mater. 2013;61:6577.

[19] Kuhlmanwilsdorf D, Hansen N. Scr.Metall.Materialia 1991;25:1557.

[20] Christiansen G, Bowen JR, Lindbo J. Mater Charact 2002;49:331.

[21] Liu Q. Ultramicroscopy 1995;60:81. 
[22] Yu, T, Hansen, N, Huang, X, Winther, G. Nanostructured Metals: Fundamentals to Applications 2009:393.

[23] Nes E. Acta Metall.Mater. 1995;43:2189.

[24] Yu T, Hansen N, Huang X. Philos.Mag. 2012;92:4056.

[25] Albou A, Borbely A, Maurice C, Driver JH. Philosophical Magazine 2011;91:3981.

[26] Gottstein G, \& Shvindlerman LS. Grain boundary. 2010.

[27] Jazaeri H, Humphreys FJ, Bate PS. Aluminium Alloys 2006, Pts 1 and 2 - Research through Innovation and Technology 2006;519-521:153.

[28] Read WT, Shockley W. Physical Review 1950;78:275.

[29] Huang Y, Humphreys FJ. Acta Mater. 2000;48:2017.

[30] Deng Shanquan, Godfrey A, Mishin O.V., Hansen Niels, Huang Xiaoxu. 2014:251. 